\title{
Shaping the Future of Patient Experience Measurement in Canadian Healthcare
}

\section{-}

\author{
INTRODUCTION
}

Jason M. Sutherland, $\mathrm{PHD}$

Associate Professor, Centre for Health Services and Policy Research

Program Head, Health Services and Outcomes, Centre for Health Evaluation \& Outcome Sciences

School of Population and Public Health, Faculty of Medicine, University of British Columbia

Scholar, Michael Smith Foundation for Health Research

Vancouver, BC

Patients' experiences with healthcare providers can be tumultuous and stressful. Focusing on what matters, the experience of care is important to improving patients' quality of life. In this issue's lead article, Kuluski and colleagues (2017) propose moving beyond setting-based patient experience measures. In doing so, the authors emphasize the importance of caregivers and the communities in which patients live in improving patients' and caregivers' care experiences. To achieve these aims, however, the authors demand a higher level of reflexivity from provinces' healthcare funders and providers.

Kuluski and colleagues articulate a vision for expanding the measurement and prominence of healthcare experiences of patients and their families. As patients' experiences change over time, settings and treatments, the authors propose measuring care experience across the continuum of care, including outside formal healthcare structures. However, the authors note that, for new care experience measures to be effective, patients' providers will have to act on them.

All authors in this issue support the thesis that the collection, reporting and use of patients' and caregivers' experiences with their healthcare should be greatly expanded. To be successful, new measures must provide meaningful information to patients, caregivers and their providers, with the expectation 
of improving care processes and quality of life across the continuum of care (Estabrooks 2017). Meaningfulness should accelerate the science of measuring care experiences, as there are too few validated and meaningful instruments that span the continuum of care (McCloskey 2017; Quaglietta et al. 2017). It should also accelerate the question of what to measure, as there is no consensus of what is important to measure as healthcare delivery evolves over time (Doupe 2017).

The benefits of measuring care experience to improve quality of life are compelling and clearly noted by Nuti et al. (2017) and Glasby (2017), wherein closing the feedback loop between providers with patients' care experiences identify quality or safety problems and risky gaps in care (Murante 2014; Noest 2014). For health systems, Nuti et al. (2017) describe how aggregated experience measures can form an element of integrated performance measurement for care pathways. The Toronto Central Local Health Integration Network's successful efforts demonstrate that a region can prioritize experience measures in Canada (Wojtak 2017). In complementary work, McCloskey et al. (2017) propose that geriatric medicine, through its linkages across care teams and settings, and underpinned by primary care, is a strong starting point for measuring cross-continuum care experience.

As emphasized by Wong et al. (2017), however, securing providers' support and engagement for improving patients' care experience will be complex and multi-factorial - including new infrastructure for collecting care experience data that doesn't complicate providers' workflow, addresses differences in cultural perception of care experience, minimizes patients' burden and overcomes limits of English-language instruments. Innovative solutions to these technical challenges could be generated by targeting national health research funding priorities on these issues.
... how should provincial funders incorporate these additional measures?

Technical and financial creativity will be required to address new workload demands - finding new time for providers to interpret, and act on, new streams of patients' care experience data. To counter the challenge of our complex and reactive healthcare environments, Kuluski et al. (2017) recommend that care experience be nested within engagement-capable environments. However, as noted by Estabrooks (2017), provinces' current structures and processes appear to have limited capability for engaging with, and changing, providers' behaviours to adapt to patients' and caregivers' experiences with their environment.

Sustained financial resources will be required to surmount the barriers described above. This presents opportunities for federal organizations to partner with provinces, regions, and providers (Wojtak 2017). Technical solutions - possibly electronic medical record-based - will need to be developed to collect, secure and link comparable care experience measures across the care continuum. However, Doupe's (2017) article points out two unanswered questions regarding aggregating and reporting care experience information. First, as there are already many system-level performance measures, how should provincial funders incorporate these additional measures? Second, how should provinces evaluate the effectiveness of measuring care experience and demonstrate that they have improved healthcare by responding to patient experience measures?

There is no doubt that measuring what matters to patients will lead to improving patients' and caregivers' quality of life. 
Although the papers in this issue are framed in the context of Canadian healthcare, the importance and relevance of comprehensive measurement of care experience is important in many other countries. The authors also provide a starting point for answering how care experience measures may be used to generate policy options for system-level healthcare challenges. Based on the opportunities described by authors in this issue, a broad coalition of patients, providers and healthcare funders is now needed for moving this agenda forward.

\section{References}

Doupe, M. 2017. "More Than Just Measurement." Healthcare Papers 17(2): 46-49. doi:10.12927/ hcpap.2017.25410.

Estabrooks, C.A. 2017. "Engagement Capable Environments: No Less Challenging than Other Large System Change." Healthcare Papers 17(2): 40-45. doi: 10.12927/hcpap.2017.25411.

Glasby, J. 2017. "Health System Performance Measurement: A UK Perspective.” Healthcare Papers 17(2): 30-33. doi:10.12927/hcpap.2017.25413.

Kuluski, K., M.L.A. Nelson, C.S. Tracy, C.A. Alloway, C. Shorrock, S. Shearkhani et al. 2017. "Experience of Care as a Critical Component of Health System Performance Measurement: Recommendations for Moving Forward." Healthcare Papers 17(2): 8-20. doi:10.12927/hcpap.2017.25415.
McCloskey, R., P. Jarrett and L. Yetman. 2017. "Putting Performance Measurement Recommendations into Practice: Building on Current Practices." Healthcare Papers 17(2): 65-71. doi:10.12927/hcpap.2017.25407.

Murante, A.M., M. Vainieri, D.C. Rojas and S. Nuti. 2014. "Does Feedback Influence Patient - Professional Communication? Empirical Evidence from Italy." Health Policy 116(2-3): 273-80.

Noest, S., S. Ludt, A. Klingenberg et al., 2014. "Involving Patients in Detecting Quality Gaps in a Fragmented Healthcare System: Development of a Questionnaire for Patients' Experiences Across Health Care Sectors (PEACS)." International Journal for Quality in Health Care 26(3): 240-49.

Nuti S., S. De Rosis, M. Bonciani and A. Murante. 2017. "Rethinking Healthcare Performance Evaluation Systems towards the People-Centeredness Approach: Their Pathways, their Experience, their Evaluation." Healthcare Papers 17(2): 56-64. doi:10.12927/hcpap.2017.25408.

Quaglietta, J. and K. Popovich. 2017. "Experience of Care - Furthering the Patient Experience Agenda." Healthcare Papers 17(2): 50-55. doi:10.12927/ hcpap.2017.25409.

Wojtak, A. 2017. "Reacting to Patient Experience Results - How Can We Be Proactive?" Healthcare Papers 17(2): 34-49. doi:10.12927/hcpap.2017.25412.

Wong, S., S. Johnston, F. Burge, K. McGrail and W. Hogg. 2017. "Measuring Patient Experiences: Is It Meaningful and Actionable?" Healthcare Papers 17(2): 22-29. doi:10.12927/hcpap.2017.25414.

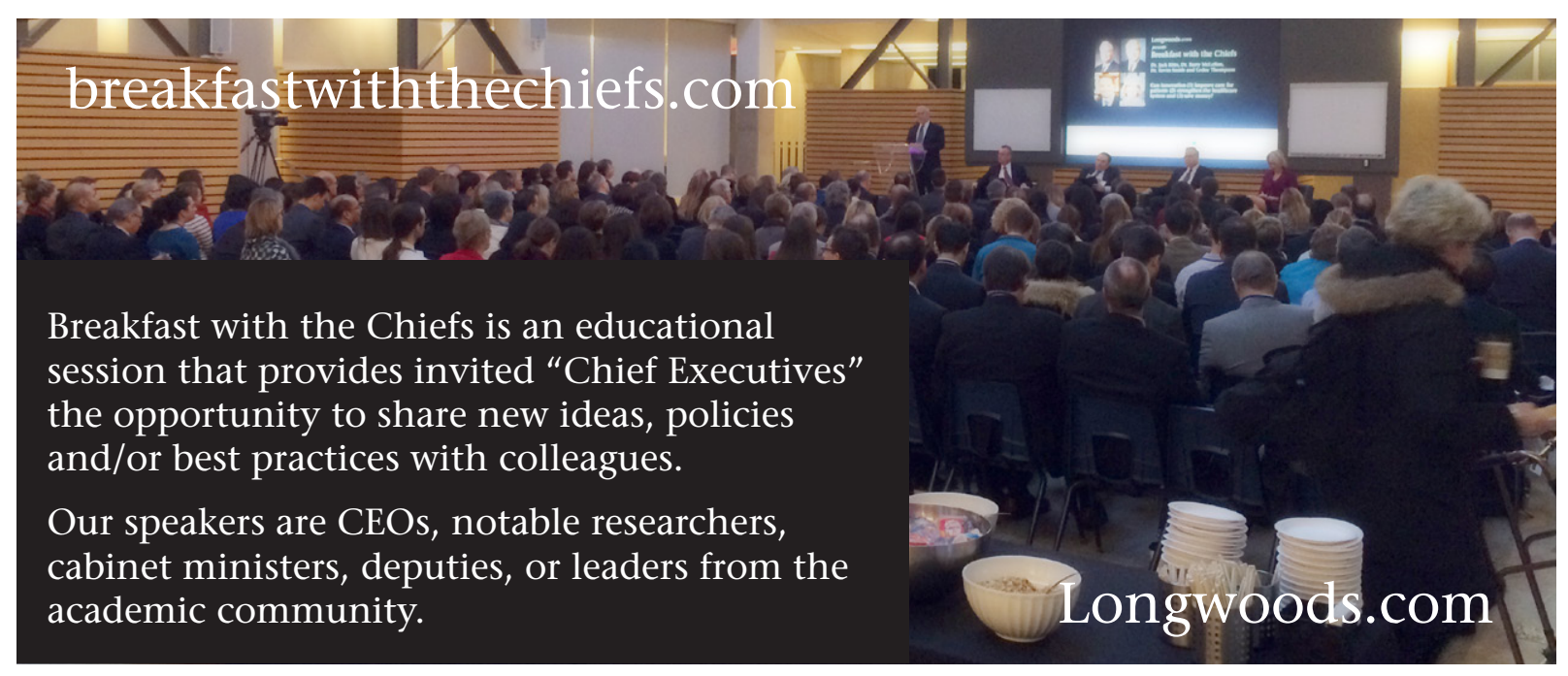

